\section{Evaluación del tratamiento de linfoma de Hodgkin con esquema ABVD en Chile}

\author{
MARÍA ELENA CABRERA C. ${ }^{1}$, BÁRBARA PUGA L. ${ }^{2}$, \\ VIVIANNE TORRES ${ }^{3}$, MAURICIO SALINAS ${ }^{4}$
}

\section{Treatment of Hodgkin lymphoma. Analysis of 915 patients}

Background: Hodgkin lymphoma has a high rate of curability, even in advanced stages. Aim: To assess the results of Hodgkin lymphoma treatment using the $A B V D$ (doxorubicin, bleomycin, vinblastine, dacarbazine) chemotherapy regimen. Material and Methods: Analysis of a database held by the Chilean Ministry of Health, including all patients treated at accredited cancer treatment centers. Results: Data for 915 patients, median age 35 years (range 15-86 years) and followed for a median of 97 months (range 1-347 months) were analyzed. Forty-one percent had localized disease. Overall survival at five years for localized and advanced stages was $92 \%$ and $74 \%$, respectively. The figures for progression free survival were $87 \%$ and $64 \%$, respectively. Patients with relapse who received autologous stem cell transplantation (ASCT) had a five year overall survival of $92 \%$, compared to $64 \%$ among those who did not undergo this procedure $(p<0.01)$. The Guarantees in Health Program set up by the Ministry of Health, was associated with earlier stage disease at diagnosis. Conclusions: The ABVD regimen achieves high rates of cure in localized stages of the disease but the results in advanced stages are not optimal. ASCT significantly improves survival in patients with relapse. The Guarantees in Health Program is associated with earlier diagnosis of the disease.

(Rev Med Chile 2019; 147: 437-443)

Key words: Drug Therapy; Hodgkin Disease; Stem Cell Transplantation; Survival.

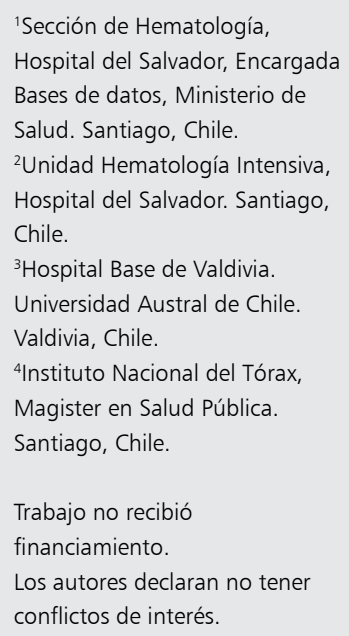

Recibido el 28 de noviembre de 2018, aceptado el 16 de abril de 2019.

Correspondencia a: Dra. María Elena Cabrera C. Universidad de Chile Sección de Hematología Hospital del Salvador Av. Salvador 364, Santiago, Chile. mecabrera.1950@gmail.com
E 1 linfoma de Hodgkin (LH) constituye alrededor de $15 \%$ de los linfomas y afecta principalmente a personas jóvenes. En Chile la incidencia es de $0,52 \times 100.000$ habitantes $^{1}$. El tratamiento actual logra tasas de sobrevida entre 90-96\% en etapas localizadas ${ }^{2,3}$ y $80-95 \%$ en etapas avanzadas ${ }^{4,5}$. Los desafíos actuales son reducir los efectos tóxicos tardíos del tratamiento, particularmente en pacientes en etapas precoces de buen pronóstico, en los cuales, la causa de muerte por complicaciones relacionas al tratamiento, segun- das neoplasias y enfermedad cardiovascular, excede a la recaída del linfoma, 15 a 20 años después ${ }^{6-8}$.

Por este motivo el objetivo de los tratamientos de las últimas décadas ha sido reducir la intensidad del tratamiento en pacientes con enfermedad localizada, disminuyendo los campos y dosis de radioterapia $(\mathrm{RT})^{2,3}$, excluirla completamente ${ }^{9,10}$ o disminuir el número de ciclos de quimioterapia $(\mathrm{QT})^{3}$, y por otro lado, intensificar el tratamiento de primera línea en pacientes de alto riesgo con enfermedad avanzada ${ }^{5,11,12}$. 
El tratamiento estándar en la actualidad, continúa siendo la quimioterapia $\mathrm{ABVD}$, asociada a $\mathrm{RT}$ en etapas precoces ${ }^{13,14}$. El esquema BEACOPP escalado, produce un mejor control inicial de la enfermedad, sin embargo, la sobrevida a largo plazo es la misma, con un mayor costo en toxicidad a corto y largo plazo ${ }^{5,11,12}$. En caso de enfermedad refractaria y recaída, el estándar es proceder a terapia de rescate y TPH autólogo, capaz de curar $30 \%$ y $65 \%$ de los pacientes, respectivamente ${ }^{14,15}$.

El linfoma fue incorporado al Régimen de Garantías Explicitas de Salud (GES) en Chile el año 2005, lo que significa garantías de acceso, oportunidad y cobertura financiera, en el Sistema de Salud de Chile, público y privado.

El presente estudio describe los resultados de 915 pacientes tratados con el esquema ABVD, en el sistema público chileno, incluyendo 167 pacientes, también incluidos en una publicación previa ${ }^{16}$. El objetivo del presente trabajo fue evaluar los resultados de este tratamiento, con mayor número de casos y seguimiento más prolongado, y evaluar el impacto de la inclusión del linfoma de Hodgkin en el Programa GES.

\section{Material y Método}

Se incluyó todos los pacientes mayores de 15 años, con diagnóstico de Linfoma de Hodgkin, registrados en la base de datos del Ministerio de Salud y tratados en los centros acreditados para el tratamiento de cáncer del adulto PANDA, del sistema público de salud. Se excluyó pacientes con serología para virus de inmunodeficiencia humana (VIH) positiva. El diagnóstico patológico fue realizado en un laboratorio de referencia (Dra. Virginia Martínez) en 78\% de los casos. El resto, en los hospitales respectivos. La etapa clínica se determinó en base a la historia clínica y examen físico; hemograma y VHS, perfil bioquímico, radiografía de tórax, tomografía axial computada (TAC) de tórax, abdomen y pelvis y biopsia ósea de cresta ilíaca.

El protocolo para etapas localizadas (I y II) consistió en 4 ciclos ABVD asociada a RT de campos comprometidos 30 Gy y para etapas avanzadas (III y IV) 6-8 ciclos ABVD. Si había masa bulky $(>10 \mathrm{~cm})$ se asociaba RT. Se evaluó la respuesta después del $3^{\circ}$ ciclo con TAC. El PET/CT se utilizó ocasionalmente, cuando estuvo disponible, prin- cipalmente para la evaluación de masas residuales. Los criterios de respuesta se definieron de acuerdo al Consenso Internacional para Linfoma ${ }^{17,18}$. Remisión completa (RC): desaparición de toda evidencia de enfermedad, remisión parcial (RP): disminución de $\geq 50 \%$ de las masas, enfermedad estable (EE): disminución $\leq 50 \%$ de las masas y recaída (después de $\mathrm{RC}$ )/progresión (después de $\mathrm{RP} / \mathrm{EE}$ ): aparición de nuevas lesiones o aumento $\geq 50 \%$ de las masas existentes. Los pacientes se controlaron cada 4 meses el primer año, cada $6 \mathrm{del}$ segundo al quinto año y anualmente de por vida. Los pacientes que fueron refractarios o recayeron, recibieron esquema de rescate ESHAP (etopósido, cisplatino, metilprednisolona, citarabina) y si no hubo respuesta, esquema ICE (ifosfamida, carboplatino, etopósido). Luego se realizó TPH en menores de 40 años, acondicionado con régimen BEAM (carmustina, etopósido, citarabina, melfalán) según las indicaciones del Protocolo de Trasplante del PANDA. Todos los pacientes firmaron consentimiento informado.

\section{Análisis estadísticos}

La sobrevida global (SG) fue medida desde la fecha de diagnóstico hasta la muerte por cualquier causa o vivo al último seguimiento (censado). La sobrevida libre de progresión (SLP) fue medida desde la fecha de diagnóstico hasta la fecha de recaída, progresión o muerte por cualquier causa o vivo en remisión completa (RC) al último seguimiento (censado). Se construyeron curvas de Kaplan y Meier para SG y SLP y prueba de log rank para comparar grupos. Se calculó intervalos de confianza de $95 \%$. Se consideró significativo $\mathrm{p}<0,05$. Los datos fueron procesados en el programa estadístico STATA versión 13.0.

\section{Resultados}

Se evaluaron 915 pacientes, tratados entre 1990-2014, 167 casos del período 1990-2003 y 748 del período 2004-2014, en los siguientes centros: Hospital del Salvador 162, Barros Luco Trudeau 133, Instituto Nacional del Cáncer 118, Sótero del Río 108, San Juan de Dios 81, San Borja Arriarán 69, Talca 67, Valdivia 53, La Serena 27, Gustavo Fricke 21, Talcahuano 19, Van Buren 14, Osorno 14, Arica 12, Concepción 6, Antofagasta 5, Temuco 5, Punta Arenas 1. Las características clínicas se 
presentan en la Tabla 1. El 55,7\% eran mujeres. No se observó aumento de casos en > 60 años. En 598 casos $(65 \%)$ se consignó la presencia de síntomas B. El compromiso extraganglionar fue en: médula ósea 117 pacientes, pulmón y pleura 88 e hígado en 64. Se observó una asociación entre sexo masculino y presentación en etapas avanzadas (59\%) e histología celularidad mixta (47\%). En cambio

Tabla 1. Características de los pacientes con Linfoma de Hodgkin $(\mathbf{n}=915)$

\begin{tabular}{|lc|}
\hline Características al diagnóstico & n (\%) \\
\hline Relación H/M & $1 / 1,26$ \\
\hline Edad media, años (rango) & $35(15-86)$ \\
$>\quad 60$ años & $154(16,8)$ \\
\hline Histología & \\
Nodular predominio linfocítico & $18(2)$ \\
Clásico esclerosis nodular & $408(45)$ \\
Clásico celularidad mixta & $407(44)$ \\
Clásico rico en linfocitos & $30(3)$ \\
Clásico depleción linfocitaria & $52(6)$ \\
Etapa clínica & \\
I & $50(5)$ \\
II & $331(36)$ \\
III & $256(28)$ \\
IV & $278(30)$ \\
\hline Síntomas B & $598(65)$ \\
Médula ósea positiva & $117(13)$ \\
\hline Etapa localizada & $381(41,6)$ \\
Etapa avanzada & $534(58,4)$ \\
\hline
\end{tabular}

en las mujeres, la asociación con presentación en etapas localizadas (48\%) e histología esclerosis nodular $(50 \%)$, fue menor que en el estudio previo. El seguimiento medio fue 97 meses, 8 años (rango, 1-347 meses). Ciento sesenta y siete casos, corresponden a pacientes incluidos en el trabajo publicado previamente.

\section{Respuesta al tratamiento}

Los resultados del tratamiento se observan en la Tabla 2. De los 71 pacientes en etapas localizadas con respuesta parcial/refractario (RP/R), 55 (77\%) recibieron QT de rescate, la mayoría (94\%) ESHAP y 19 se consolidaron con TPH. Más de la mitad (57\%) recibió RT complementaria. De los 197 casos con RP/R en etapas avanzadas, 110 (56\%) recibieron QT de rescate, la mayoría (95\%) ESHAP y 30 se consolidaron con TPH. Un tercio $(33 \%)$ recibió RT complementaria por masa bulky.

Tabla 2. Resultados del tratamiento con ABVD ( $n=915)$

\begin{tabular}{|lcc|}
\hline & $\begin{array}{c}\text { Etapas } \\
\text { localizadas } \\
\mathbf{n = 3 8 1}(\%)\end{array}$ & $\begin{array}{c}\text { Etapas } \\
\text { avanzadas } \\
\mathbf{n = 5 3 4}(\%)\end{array}$ \\
\hline Remisión completa & $310(81,4)$ & $337(63)$ \\
\hline Remisión parcial/refractario & $71(18,6)$ & $197(37)$ \\
\hline SG a 5 años & 91,7 & 74 \\
\hline SLP a 5 años & 87,2 & 64,3 \\
\hline
\end{tabular}

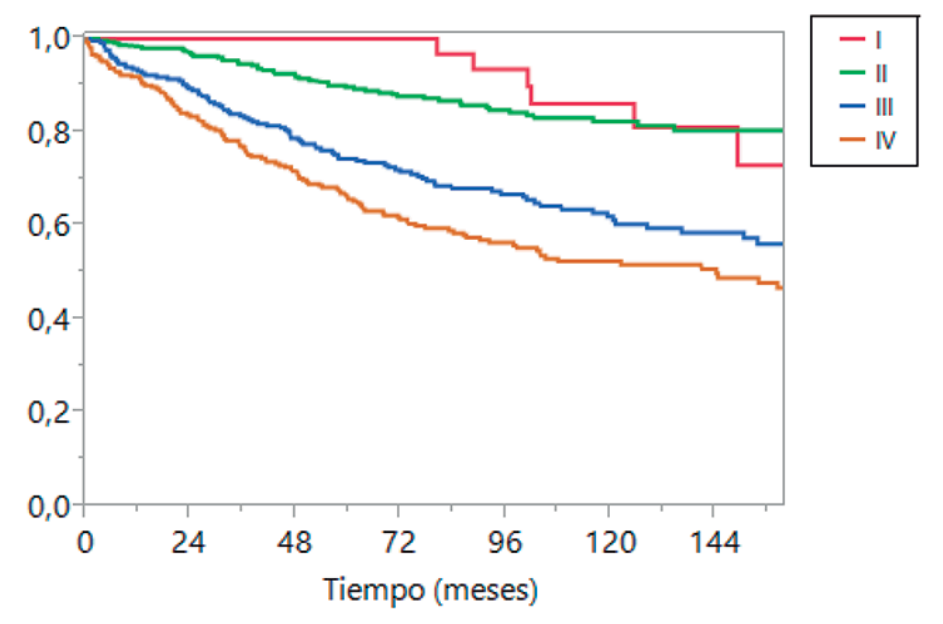

Figura 1. Sobrevida global de acuerdo a la etapa clínica. 


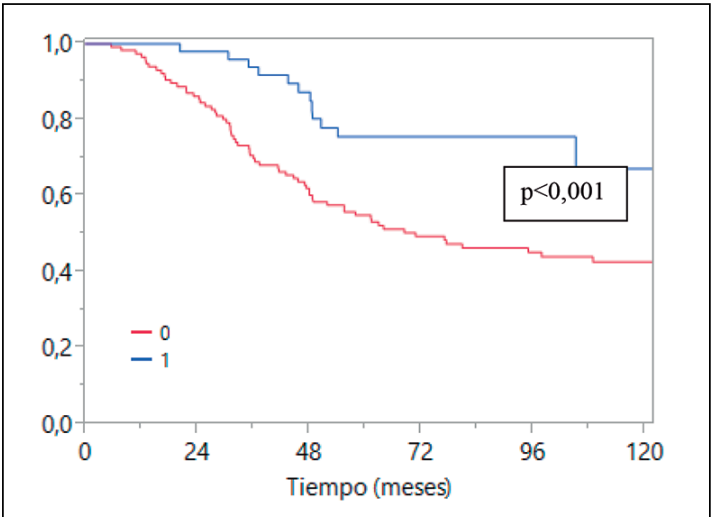

Figura 2. Sobrevida global de pacientes con Linfoma de Hodgkin y respuesta parcial/refractario que recibieron trasplante autólogo y no lo recibieron.

Ciento once pacientes han recaído, 95 fueron tratados con QT de segunda línea, de ellos 67 recibieron solo QT y 28 se consolidaron con un TPH.

\section{Sobrevida}

A 5 años, la SG de la etapa clínica I, II, III y IV fue $100 \%, 90,5 \%, 75,5 \%$ y $66,6 \%$, respectivamente (Figura 1) y la SLP fue 100\%, 85,2\%, 69,4\% y $59,6 \%$, respectivamente. Agrupadas por etapas localizadas y avanzadas, la SG fue $91,7 \%$ y $74 \%$ y la SLP fue $87,2 \%$ y $64,3 \%$, respectivamente.

La SG a 5 años de todos los pacientes con RP/ refractarios (116/165) que recibieron solo QT fue $54,9 \%$, en cambio aquellos que se consolidaron con un TPH (49/165) fue 75,6 \% (p < 0,01). (Figura 2).A su vez, la SG a 5 años de los pacientes en recaída que recibieron solo QT de rescate (67/95) fue $64,1 \%$, en cambio aquellos que se consolidaron con un TPH $(28 / 95)$ fue $91,7 \%(\mathrm{p}<0,01)$. (Figura 3). Una paciente recayó, 9 años post TPH.

\section{Toxicidad}

Respecto a efectos adversos, se observó toxicidad a la bleomicina en seis pacientes $(0,65 \%)$, cinco de los cuales eran mayores de 55 años, dos con antecedentes de tabaquismo y otro de asma. Cinco fallecieron (mortalidad del total de casos tratados $0,5 \%$ ). Entre los efectos adversos tardíos, se observó 10 casos de segunda neoplasia (1\%): dos casos cáncer de pulmón, dos linfoma no Hodgkin (difuso células grandes B y MALT gástrico), leucemia mieloide aguda, leucemia

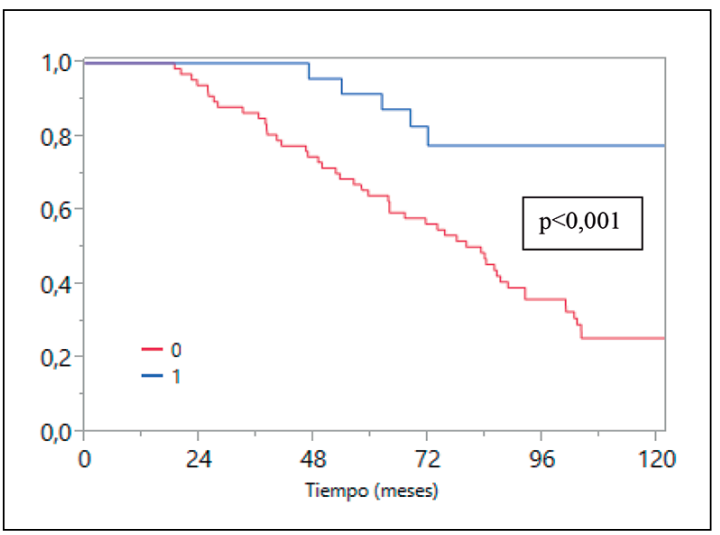

Figura 3. Sobrevida global de pacientes con Linfoma de Hodgkin en recaida que recibieron trasplante autólogo y no lo recibieron.

linfoblástica aguda, mesotelioma peritoneal y melanoma, un caso cada uno. Nueve casos fallecieron por cardiopatía coronaria (1\%), tres de ellos con irradiación de mediastino.

\section{Programa GES}

Se observó que en el período previo al inicio del programa GES (1990-2004), 38\% (120/316) se diagnosticaba en etapa localizada, en cambio en el período posterior (2005-2014), 43,5\% (261/599) se diagnosticó en etapa limitada.

\section{Discusión}

La evaluación de los pacientes con linfoma de Hodgkin tratados con ABVD en la red del sistema público de salud de Chile, muestra una mejoría de la sobrevida especialmente en las etapas localizadas, respecto de la publicación previa. Es así como la $S G$ a 5 años de los pacientes tratados con ABVD en etapas localizadas, mejoró de $85 \%$ a $91,7 \%$, con respecto al período anterior, aunque esta no fue significativa $(p=0,27)$. En este grupo se incluyó también casos con etapa IIB bulky, casos que son excluidos en la mayoría de los estudios. En cambio, en las etapas avanzadas, la SG a 5 años fue incluso menor, $76 \%$ y $71 \%$, respectivamente. Las razones de la falta de mejoría en este grupo, podría deberse a un seguimiento medio mucho mayor en el estudio actual, prácticamente el doble, 97 y 49 meses, respectivamente y que este estudio incluye un 
número de pacientes considerablemente mayor, 915 y 167 casos, respectivamente. En la literatura la sobrevida de las etapas avanzadas sobrepasa $80 \%$. Por este motivo, puede plantearse realizar tratamientos adaptados a la respuesta, como iniciar la QT con ABVD y luego escalar a otro esquema, ESHAP, ICE o BEACOPP, si el PET/CT es positivo después de dos ciclos. También se podría iniciar la QT con esquemas de segunda línea en pacientes de alto riesgo, con score pronóstico internacional mayor a 4 puntos, en pacientes menores de 60 años.

Las características epidemiológicas en cuanto a subtipos histológicos, no ha mostrado variación respecto al estudio previo, con una distribución similar entre esclerosis nodular y celularidad mixta, a pesar de la mejoría del nivel socioeconómico de la población chilena ${ }^{19}$. Al igual que en el estudio previo, se observó que los varones se presentan en etapas más avanzadas y con histología celularidad mixta. En cambio en el sexo femenino, la asociación con etapas localizadas e histología esclerosis nodular, fue menos evidente.

El esquema ABVD está asociado a efectos adversos aceptables. Sin embargo, la exposición a bleomicina conlleva el riesgo de efectos tóxicos pulmonares serios, incluso fatales. En nuestro estudio la frecuencia fue $0,6 \%$ y mortalidad $0,5 \%$. La literatura describe una toxicidad grado 3 o 4 de $5 \%-10 \%$ y mortalidad (del total de pacientes tratados) de $1 \%$ a $3 \%{ }^{20,21}$. Como los síntomas son inespecíficos, disnea, fiebre o tos, es muy probable que nuestras cifras bajas se deben a un sub diagnóstico. El riesgo aumenta con la edad, especialmente en mayores de 40 años, con antecedentes de tabaquismo y enfermedades pulmonares como asma o bronquitis crónica, función renal disminuida y RT de mediastino. Por tanto, se recomienda identificar pacientes con factores de riesgo, antes de iniciar la QT, monitorear los síntomas de toxicidad pulmonar y suprimirla en quienes tienen antecedentes de daño pulmonar ${ }^{22}$. Un estudio reciente ${ }^{23}$, omitió la bleomicina en pacientes con enfermedad avanzada y PET interino negativo después de dos ciclos ABVD, sin disminuir la eficacia y con menos efectos tóxicos pulmonares (SLP a 3 años $85,7 \%$ vs $84,4 \%$, con y sin bleomicina, respectivamente).

Se observó que la disponibilidad del TPH para pacientes con enfermedad refractaria/recaida, significó una mejoría significativa de la sobrevida, en comparación con aquellos que no lo recibieron.
Esta alternativa terapéutica está disponible para los pacientes del sistema público de salud de Chile desde 2004, y el acceso es expedito.

Se observó una asociación entre la implementación del Programa GES y un diagnóstico más precoz del linfoma de Hodgkin en Chile, el año 2005.

La mejoría en la sobrevida observada en este estudio con respecto a la publicación previa en pacientes en etapas localizadas, se debe a un mayor acceso a la salud, detección más precoz de la enfermedad, $y$ a otros factores, tales como mayor experiencia del equipo de salud, disponibilidad de esquemas de rescate y mayor acceso al TPH.

El uso de la tomografía con emisión de positrones (PET/CT) está siendo utilizada en los últimos años para realizar "tratamientos adaptados a la respuesta"13-15. Por ejemplo, en pacientes con etapa localizada no bulky, si el PET es negativo después de tres ciclos ABVD, la RT puede omitirse, y la SLP y SG son similares, con o sin $\mathrm{RT}^{24}$. A su vez, en pacientes con etapa avanzada, un PET negativo después de dos a cuatro ciclos de ABVD, predice una evolución favorable. En cambio un PET positivo, se beneficiaría de un tratamiento intensificado ${ }^{25-27}$.

Más recientemente, el conocimiento de la biología molecular de la enfermedad ha llevado al desarrollo de agentes altamente efectivos, como el anticuerpo conjugado a droga, brentuximab vedotin, anti CD30, antígeno expresado en la célula de Reed-Sternberg, utilizado en casos de $\mathrm{LH}$ refractario o incluso incorporado a regímenes de primera línea en forma experimental ${ }^{13-15} \mathrm{y}$ drogas que bloquean células $\mathrm{T}$ intratumorales que impiden la muerte celular programada, anti PD- $1^{28}$.

En resumen, el presente estudio demuestra una mejoría en la sobrevida de las etapas localizadas de la enfermedad, en comparación con la década anterior y una asociación con diagnóstico más precoz, desde el inicio del programa GES. La incorporación del trasplante autólogo, ha sido un aporte valioso, al tratamiento de pacientes con enfermedad refractaria o recaída. El tratamiento adaptado a la respuesta incorporando el PET/CT rutinariamente, sería de utilidad para mejorar el pronóstico de todos los pacientes.

Agradecimientos: A todos los médicos y equipo de salud, que han estudiado y tratado estos pacientes. 
A la Dra. Virginia Martínez, por realizar la mayoría de los estudios histopatológicos. Al Sr. Gabriel Cavada, PhD. Bioestadística, Escuela de Salud Pública, Universidad de Chile, por los análisis estadísticos iniciales.

Hematólogos Centros PANDA: (por orden en número de pacientes)

M. Elena Cabrera, Ana María Gray, Carolina Guerra, Javiera Molina, Camila Peña, Bárbara Puga, Ximena Valladares, M. Soledad Undurraga, Hospital del Salvador; Vivianne Lois, Lina Muñoz, Isabel Bustos, Hospital Barros Luco Trudeaux; Carmen Cao, Tomasa Martínez, Instituto $\mathrm{Na}$ cional del Cáncer; Hernán Rojas, Denis Suárez, Hospital Sótero del Río; Carmen Gloria Vergara, Valeska Vega, Hospital San Juan de Dios; Ricardo Hojas, Alvaro Pizarro, Hospital San Borja Arriarán; Augusto Aspillaga, Hospital Regional de Talca; Alvaro León, Susana Calderón, Lilian Pilleux, Blas Lesina, Hospital Regional de Valdivia; Ninette Blanchard, Ricardo Vacarezza, Hospital La Serena; Carlos Merino, Christine Rojas, Hospital Gustavo Fricke; Jacqueline Oliva, Alejandro Leal, Hospital de Talcahuano; Bernardita Rojas, Ximena Huerta, Hospital Van Buren; Jeanette Rosas, M. Luisa González, Hospital Regional de Osorno; Cecilia Hales, Hospital de Arica; Mónica Romero, Fernando Ibieta, Mauricio Chandía, Hospital Regional de Concepción; Marjorie Gill, Hospital de Antofagasta, Mónica Moncada, Marisa Capurro, Hospital de Temuco; Bogdam Liberon, Hospital de Punta Arenas.

\section{Referencias}

1. Globocan 2018: Estimated Cancer Incidence, Mortality and Prevalence Worlwide in 2018. http://globocan.iarc. $\mathrm{fr} /$ Pages/fact_sheets_population.aspx.

2. Bonadonna G, Bonfante V, Viviani S, Di Russo A, Villani F, Valagussa P. ABVD plus subtotal nodal versus involved-field radiotherapy in early-stage Hodgkin's disease: Long term results. J Clin Oncol 2004; 22: 283541.

3. Engert A, Plutschow A, Eich HT, Lohri A, Dörken B, Borchmann P, et al. Reduced treatment intensity in patients with early-stage Hodgkin's lymphoma. N Engl J Med 2010; 363: 640-52.

4. Duggan DB, Petroni GR, Johnson JL, Glick JH, Fisher RI, Connors JM, et al. Randomized comparison of ABVD and MOPP/ABV hybrid for the treatment of advanced Hodgkin's disease: Report of an intergroup trial. J Clin Oncol 2003; 21: 607-14.

5. Federico M, Luminari S, Iannitto E, Polimeno G, Marcheselli L, Montanini A, et al. HD2000 Gruppo Italiano per lo Studio dei Linfomi Trial. ABVD compared with BEACOPP compared with CEC for the initial treatment of patients with advanced Hodgkin's lymphoma: Results from the HD2000 Gruppo Italiano per lo Studio dei Linfomi Trial. J Clin Oncol 2009; 27: 805-11.

6. Armitage JO. Early-Stage Hodgkin's Lymphoma. N Engl J Med 2010; 363: 653-62.

7. Castellino SM, Geiger AM, Mertens AC, Leisenring WM, Tooze JA, Goodman P, et al. Morbidity and mortality in long-term survivors of Hodgkin lymphoma: a report from the Childhood Cancer Survivor Study. Blood 2011; 117: 1806-16.

8. Ng AK. Review of the cardiac long-term effects of therapy for Hodgkin Lymphoma. Br J Haematol 2011; 154: 23-31.

9. Meyer RM, Gospodarowicz MK, Connors JM, Pearcey RG, Wells W, Winteret JN, et al. ABVD alone versus radiation-based therapy in limited-stage Hodgkin's lymphoma. N Engl J Med 2012; 366: 399-408.

10. Yahalom J. Chemotherapy only in early-stage Hodgkin lymphoma: More relapses but "same" (or possibly worse) survival. Reconsidering the misguided trend to omit radiotherapy. Curr Hematol Malig Rep 2014; 9: 212-6.

11. Diehl V, Franklin J, Pfreundschuh M, Lathan B, Paulus $\mathrm{U}$, Hasenclever D, et al. Standard and increased-dose BEACOPP chemotherapy compared with COPP-ABVD for advanced Hodgkin's disease. N Engl J Med 2003; 348: 2386-95.

12. Engert A, Diehl V, Franklin J, Lohri A, Dörken B, Ludwig WD, et al. Escalated-dose BEACOPP in the treatment of patients with advanced-stage Hodgkin's lymphoma: 10 years of follow-up of the GHSG HD9 study. J Clin Oncol 2009; 27: 4548-54.

13. Bartlett NL. Fine tuning the treatment of Hodgkin's lymphoma. Editorial. N Engl J Med 2016; 374: 2490-2.

14. Townsend W, Linch D. Hodgkin's lymphoma in adults Lancet 2012; 380: 836-47.

15. Ansell SM. Hodgkin lymphoma: 2014 Update on diagnosis, risk-stratification, and management. Am J Hematol 2014; 89: 772-9.

16. Cabrera ME, García H, Lois V, León A, Peña K, Rossle A, et al. por el Programa Nacional de Cáncer del Adulto. Linfoma de Hodgkin en Chile. Experiencia de 15 años del programa de drogas antineoplásicas del adulto. Rev Med Chile 2007; 135: 341-50.

17. Cheson BD, Horning SJ, Coiffier B, Shipp MA, Fisher 
RI, Connors JM, et al. Report of an International Workshop to standardize response criteria for non-Hodgkin’s lymphomas. J Clin Oncol 1999; 17: 1244-53.

18. Cheson B, Pfistner B, Juweid M, Gascoyne R, Specht L, Horning S. et al. Revised response criteria for malignant lymphoma. J Clin Oncol 2007; 25: 579-86.

19. https://www.datosmacro.com/pib/chile. PIB de Chile 2018.

20. Froudarakis M, Hatzimichael E, Kyriazopoulou L, Lagos K, Periklis Pappas P, Tzakos AG, et al. Revisiting bleomycin from pathophysiology to safe clinical use. Crit Rev Oncol/Hematol 2013; 87: 90-100.

21. Shippee BM, Bates JS and Richards KL. The role of screening and monitoring for bleomycin pulmonary toxicity. J Oncol Pharm Pract 2016; 22: 308-12.

22. Johnson P and Hayley McKenzie H. How I treat advanced classical Hodgkin lymphoma. Blood 2015; 125 : 1717-23.

23. Johnson P, Federico M, Kirkwood A, Alexander Foss A, Berkahn L, Carella A, et al. Adapted treatment guided by interim PET-CT scan in advanced Hodgkin's lymphoma. N Engl J Med 2016; 374: 2419-29.

24. Radford J, Illidge T, Counsell N, Hancock B, Pettengell
R, Johnson P, et al. Results of a trial of PET-directed therapy for early-stage Hodgkin's Lymphoma. N Engl J Med 2015; 372: 1598-607.

25. Gallamini A, Hutchings M, Rigacci L, Specht L, Merli F, Hansen M, et al. Early interim 2-[18F]fluoro-2-deoxy-Dglucose positron emission tomography is prognostically superior to international prognostic score in advanced-stage Hodgkin's lymphoma: a report from a joint Italian-Danish study. J Clin Oncol 2007; 25: 3746-52.

26. Gallamini A, Rossi A, Patti C, Picardi M, Di Raimondo F, Cantonetti M, et al. Early treatment intensification in advanced-stage high-risk Hodgkin lymphoma patients, with a positive FDGPET scan after two ABVD courses-second interim analysis of the GITIL/FIL HD0607 clinical trial. Haematologica 2013; 98: 3-8.

27. Zinzani PL, Broccoli A, Gioia DM, Castagnoli A, Ciccone G, Evangelista A, et al. Interim Positron Emission Tomography Response-Adapted Therapy in Advanced-Stage Hodgkin Lymphoma: Final Results of the Phase II Part of the HD0801 Study. J Clin Oncol 2016; 34: 1376-85.

28. Maly J, Alinari L. Pembrolizumab in classical Hodgkin's lymphoma. Eur J Haematol 2016; 97: 219-27. 